\title{
Remote control of nanoparticles for customized direct drug delivery to tumors
}

A new study describes the development of remotely controlled nanoparticles that, when pulsed with an electromagnetic field, release drugs that will attack a tumor. By controlling when the electromagnetic field is applied and so when the drug is released, the nanoparticles become remote controlled.

The same team from $M$ assachusetts Institute of Technology (M IT; M A, USA) has al ready developed injectable multifunctional nanoparticles designed to flow through the bloodstream, home to tumors and clump together. Clinicians are helped in visualizing tumorsthrough M RI by clumped particles. Following this discovery of being able to see the clumped particles, the question then became: can we talk back to them?

"By controlling when the electromagnetic field is applied and so when the drug

is released, the nanopartic les become remote controlled."

This new research provides the answer: yes. The system uses nanoparticles that are superparamagnetic, which causes them to give off heat when they are exposed to a magnetic field. These particles are joined to active molecules, such as therapeutic drugs.

The particles radiate heat, which melts the tethers and releases the drugs, when they are exposed to an electromagnetic field made up of waves with frequencies of 350-400 kilohertz. These waves are perfectly safe to the human body, passing harmlessly through tissue and heating only the nanoparticles. The 'tethers' in the system are D N A strands - two D N A strands link together through hydrogen bonds that break when heated - one attached to the nanoparticle and the other to the drug. W hen heated by the electromagnetic field, the strands break apart: one remains attached to the nanoparticle whereas the other is attached to the now 'free' cargo. D ifferent frequencies or durations of electromagnetic pulses combined with the heat-sensitive tuneability of the D N A strands enable a single particle to carry different cargo simultaneously, which can be released at different times or in various combinations.

$M$ ice were implanted with a tumor-like gel saturated with nanoparticles to test the system. An implanted mouse was placed

in the well of a cup-shaped electrical

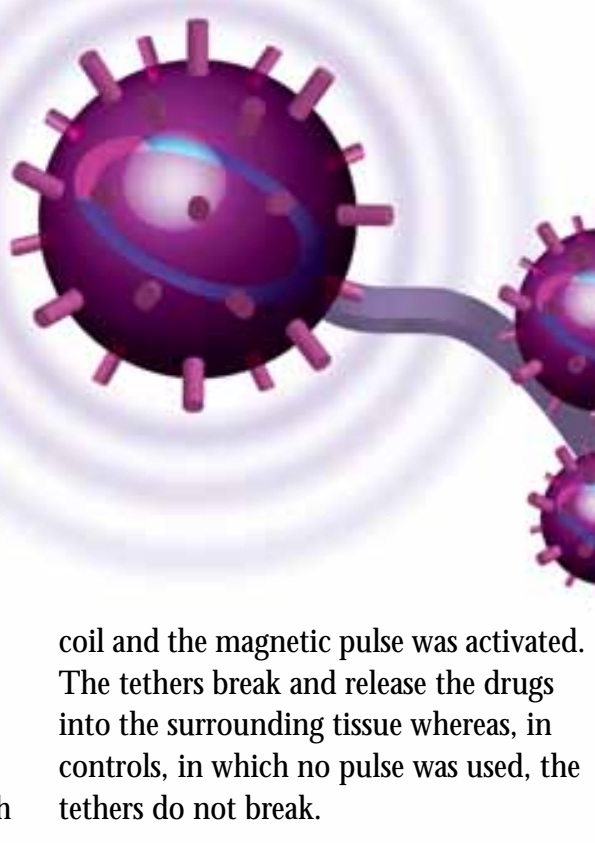

This study is a proof of concept and will be followed by more research before such therapies become viable in the clinic. Such research includes making intravenously injected nanoparticles clump together effectively enough so that a critical mass of nanoparticles, which are enough to enable the necessary region to be heated, is achieved.

"O ur overall goal is to create multifunctional nanoparticles that home to a tumor, accumulate and provide customizable remotely activated drug delivery right at the site of the disease," said Sangeeta Bhatia, lead researcher in the study.

The authors hope that this research could eventually lead to the improved diagnosis and targeted treatment of cancer.

"O ur overall goal is to create multifunctional nanoparticles that home to a tumor, accumulate and provide customizable remotely activated drug delivery right at the site of the disease."

Source: D erfus AM, von M altzahn G, H arris TJ et al.: Remotely triggered release from magnetic nanoparticles. Adv. M ater. 19(22), 3932-3936 (2007). 


\section{M ining nanodiamonds for drug delivery}

$\mathrm{N}$ anodiamonds are very effective at delivering chemotherapy drugs to cells without the negative effects associated with current drug-delivery agents, so believe a group of scientists who have reported their work recently in $\mathrm{N}$ ano Letters. Nanodiamonds will potentially have a significant role in improving cancer treatment by limiting uncontrolled exposure of toxic drugs to the body.

"T here are a lot of materials that can deliver drugs well, but we need to look at what happens after drug delivery," said $D$ ean $\mathrm{H} O$, from $\mathrm{N}$ orthwestern University's M cC ormick School of Engineering and Applied Science, who led the research. "H ow do cells react to an artificial material left in the body? $\mathrm{N}$ anodiamonds are highly ordered structures, which cells like. If they didn't, cells would become inflamed. From a patient's perspective, this is very important. And that's why clinicians are interested in our work."

The research shows that aggregated clusters of nanodiamonds are ideal for carrying a chemotherapy drug and also for shielding it from normal cells so as not to kill them, releasing the drug slowly only after it has reached its cellular target. The chemotherapy drug used in thestudy was doxorubicin hydrochloride - an apoptosis-inducing drug - and the cells used were a macrophage cell line and human colorectal carcinoma cells.

A series of genetic studies were also carried out. These analyzed three genes associated with inflammation and one gene for apoptosis in the cells used to assess the viability of the nanodiamond drug-delivery system. The nanodiamonds do not cause cell inflammation once the drug has been released and only bare diamonds are left, which is in contrast to other materials used currently for drug delivery. Inflammation is a serious side effect that can predispose a patient to cancer, block the activity of cancer drugs and even promote tumor growth. In the study, single nanodiamonds, each only 2-8 $\mathrm{nm}$ in diameter, were manipulated to form aggregated clusters of 50-100 $\mathrm{nm}$ in diameter. Thedrug is loaded onto the surface of the nanodiamonds and remains inactive within the cluster until the target is reached, the cluster breaks apart and the drug is released slowly. The large surface area of each nanodiamond cluster enables a large amount of drug to be carried and the technique provides a powerful drugrelease mechanism in a localized area.

"N anodiamonds are very special," said $\mathrm{H}$ oujin $\mathrm{H}$ uang, lead author of the paper. "They are extremely stable, and you can do a lot of chemistry on the surface, to further functionalize them for targeting purposes. In addition to functionality, they also offer safety - the first priority to consider for clinical purposes. It's very rare to have a nanomaterial that offers both."

O ther spherical nanoparticles, such as liposomes and polymersomes, used in drug delivery at present are hollow spheres filled with drug that can affect healthy cells and tissues as well as cancer cells and tumors. Although this method is effective, it can also be toxic to the healthy surrounding area. These particles are also approximately 100 times larger than nanodiamonds, which can permeate cell membranes more easily. Another clinical advantage of the nanodiamonds over many other emerging nanoparticles is that they are soluble in water.

"It's about optimizing the advantages of a material," said $\mathrm{H}$ o. "O ur team was the first to forge this area - applying nanodiamonds to drug delivery. We've talked to a lot of clinicians and described nanodiamonds and what they can do. I ask, 'Is that useful to you?' They reply, 'Yes, by all means."'

The team believe that their model could also be used for other applications, such as fighting tuberculosis or viral infection, as well as delivering cancer drugs.

"N ovel drug delivery systems, such as the one being developed by $D$ ean and his team, hold great promise in cancer therapeutics," said Steven Rosen, also from N orthwestern University. "We anticipate they will allow for more sophisticated means of targeting cancer cells while sparing healthy cells from a drug's toxicity."

Source: $H$ uang $H$, Pierstorff E, O sawa E, H o D :

Active nanodiamond hydrogels for

chemotherapeutic delivery. N ano Lett. 7(11), 3305-3314 (2007).

\footnotetext{
About the Bulletin Board

The Bulletin Board highlights some of the most important events and launches in nanomedicine and nanotechnology research. The editorial team welcomes suggestions for timely, relevant items. If you have newsworthy information, please contact:
}

Morag Robertson, PhD,

Editor, Nanomedicine,

Future Medicine Ltd, Unitec House,

2 Albert Place, London N3 1QB, UK

E-mail: m.robertson@futuremedicine.com

Tel: $+44(0) 2083716090$;

Fax: +44 (0)20 83432313 


\section{in brief...}

Modulation of tumor necrosis factor-mediated cell death by fullerenes

Harhaji L, Isakovic A, Vucicevic L et al.: Pharm. Res. DOI: 10.1007/s11095-007-9486-y (2007) (Epub ahead of print).

This study compared the ability of the fullerene $[C(60) / C(70)$ mixture-C(60/70)] nanocrystalline suspension, which was prepared by the solvent-exchange method using tetrahydrofyran [THF/nC(60/70)], with the ability of polyhydroxylated $\mathrm{C}(60 / 70)[\mathrm{C}(60 / 70)(\mathrm{OH})(\mathrm{n})]$ to modulate the cytotoxicity of the proinflammatory cytokine tumor necrosis factor (TNF). THF/nC (60/70) augmented, whereas $\mathrm{C}(60 / 70)(\mathrm{OH})(\mathrm{n})$ reduced the cytotoxicity of TNF. The numbers of cells undergoing apoptosis/necrosis were increased or reduced by $\mathrm{THF} / \mathrm{nC}(60 / 70)$ or $\mathrm{C}(60 / 70)(\mathrm{OH})(\mathrm{n})$, respectively. Oxidative stress and mitochondrial dysfunction were involved in the TNF cytotoxicity. The authors state that the ability of different fullerene preparations to modulate TNF-induced oxidative stress and subsequent cell death suggests their potential value in the TNF-based cancer therapy or prevention of TNF-dependent tissue damage.

\section{Development of lipid particles targeted via sugar-lipid conjugates as novel nuclear gene delivery system.}

Masuda T, Akita H, Nishio T et al.: Biomaterials DOI: 10.1016/j.biomaterials.2007.09.039 (2007) (Epub ahead of print).

The authors developed a novel system that mimics the mechanism of nuclear entry of adenovirus (Ad) by means of a multifunctional envelope-type nanodevice (MEND). Sugar-modified MEND had higher transfection efficiency compared with unmodified MEND in nondividing and dividing cells. Confocal microscopy confirmed that nuclear transfer of plasmid DNA (pDNA) was improved by sugar modification of M END.The study developed successfully a novel system for the nuclear delivery of pDNA by using a sugar-modified MEND and by optimizing the lipid-envelope stability.

\section{Compact cysteine-coated $\mathrm{CdSe}(\mathrm{ZnCdS})$ quantum} dots for in vivo applications.

Liu W, Choi HS, Zimmer JP, Tanaka E, Frangioni JV, Bawendi M: J. Am. Chem. Soc. 129(47), 14530-14531 (2007)

This study describes the development of a versatile nanoparticle construct using a compact cysteine coating on a CdSe(ZnCdS) core(shell) nanocrystal (QD-Cys) that is compatible biologically, very compact, highly fluorescent and functionalized easily. The QD-Cys has a small hydrodynamic diameter (approximately $6 \mathrm{~nm}$ ) enables their renal clearance in rat models. The authors believe that the ability to conjugate to QD-Cys directly provides the possibility of functionalized nanocrystals for in vivo targeted imaging, in which small targeting molecules can be appended to QD-Cys and unbound QDs can be cleared rapidly to achieve high signal:noise ratios and to reduce background toxicity.

\section{$N$ anoscale imaging agents get a versatile coat to improve clinical utility}

N ew research has found a novel nanoparticle-coating technique that uses oligonucleotides to tether targeting molecules securely to the surface of the nanoparticles. The researchers aimed to develop and characterize a flexible approach for the generation of molecular imaging agents based on gold nanoparticles conjugated to a diverse range of probe molecules. Two complementary oligonucleotide-based approaches to develop gold nanoparticle contrast agents are described.

'The researchers aimed to develop and charac terize a flexible approach for the generation of molecular imaging agents based on gold nanopartic les

conjugated to a diverse range of probe molecules.'

The study, published in Bioconjugate Chemistry and led by Rebecca Richards-Kortum (Rice University), describes a method that used sulphur-containing derivatives of DNA, which bind tightly to the surface of gold nanoparticles. A complementary D N A sequencewas used to link the D N A-coated nanoparticle to the targeting ligand. Similar imaging agents, in which targeting proteins are absorbed directly onto goldnanoparticle surfaces, are much larger than these small oligonucleotides-linked nanoparticles. The DN A tethers also result in a more stable construct under physiological conditions.

Agents were designed using this new method to detect epidermal growth factor (EGF), using either an EGF peptide or an antibody that binds to the receptor. The team al so created a contrast agent to recognize the folate receptor (which is overexpressed in many tumors) as well as one that contains a fluorescent marker. These contrast agents thus enabled multimodal imaging using both confocal reflectance imaging and standard fluorescent imaging and they also provided a significant signal boost, detected easily using the appropriate imaging technique.

The novel bioconjugation approach described in this study can be used to prepare contrast agents targeted with biomolecules that span a diverse range of sizes. It can also be adapted to develop multimodal contrast agents for molecular imaging without changing the coating design or material.

There is already tremendous promise for nanoparticles composed of metals, such as gold or iron oxide, in the field of contrast agents for molecular imaging. H owever, to turn this promise into actual clinical use requires the addition of tumortargeting molecules to the nanoparticle surface. RichardsKortum and her team believe that their new research could providea versatile method for creating such targeted nanoparticles.

Source: N itin N, Javier DJ, RichardsKortum R: O ligonucleotide-coated metallic nanoparticles as a flexible platform for molecular imaging agents. Bioconjug. Chem. 18(6), 2090-2096 (2007). 